\title{
An Exploration Of Calculating The Packet Loss Rate By Using The Block Rate
}

\author{
Fan $\mathrm{Bu}^{*}$ \\ School of Software Engineering \\ State Key Laboratory of Networking and Switching Technology Beijing University of Posts and Telecommunications \\ ${ }^{*}$ Corresponding author
}

\begin{abstract}
This electronic document is about New estimation method for link packet loss rate .An intermediate quantity is deduced from the block rate, Through this intermediate quantity to further solve the packet loss rate.
\end{abstract}

\section{Keywords—network; links; WDM; packet loss rate}

\section{INTRODUCTION}

In recent years, with the increasing expansion of computer network scale, the unstable factors in the network are increasing under the influence of equipment diversity and network complexity. At the same time, network traffic has increased sharply, and various new businesses, such as video conference, video on demand, P2P, etc., are also devouring network resources at an extremely fast speed. The measurement of network performance becomes more and more important. [1] Therefore, it is necessary to estimate the probability of information transmission instability before infrastructure construction.

Therefore, we introduce a new variable to reflect the probability of such instability, which is called the packet loss rate. Packet Loss Rate refers to the ratio of the number of packets lost in the test to the data group sent. The calculation method is: [(incoming message - output message)/ incoming message] ${ }^{*} 100 \%$. ${ }^{[2]}$

However, in practical applications, such estimation usually takes place before the construction of the entire network, so it is required that we can successfully estimate the packet loss rate by relying on some parameters in the network. In the usual calculation, direct logical calculation is used to estimate the packet loss rate of the entire network.

In some cases in our usual application, the measurement of the blocking rate may be compared with the measurement of the loss rate, so this paper tries to propose a new method to estimate the loss rate using the reverse blocking rate.

\section{Model ANALYsis}

\section{A. Selecting a Template}

A key feature of the multicast communication model is the multicast group that provides indirect identification. Neither sender nor receiver needs to know the other party's specific conditions. The sender only needs to send the packet to a multicast address and the receiver only needs to tell the network that it wants to receive the packet that sent it. Therefore, the transmission information of multicast network can be considered as the transmission of two unicast model segments. Therefore, the packet loss rate is considered as unicast model in this paper.

\section{B. Considering the Situation of link Network}

In the process of measuring the packet loss rate, The basis is to consider the current link network situation. The link network model analyzed in this paper is WDM model. We abstract it as follows, Any two nodes are connected by a link that determines the number of bars ( $\mathrm{k}$ bars). Each of these links has a certain number of ( $\mathrm{w}$ kinds) wavelengths to transmit, A wavelength converter that can be applied between partial wavelengths is set up at some of the nodes. Because of the difficulty of directly analyzing the converter with wavelength conversion, Therefore, this paper adopts the method of analyzing the condition without wavelength converter first.

\section{Reference Auxiliary Variables}

The probability of $\mathrm{k}$ paths where the wavelength is a certain value $(\lambda)$ and between two certain nodes $(i, j)$ all being blocked (This variable are recorded as $\mathrm{C}_{\mathrm{k}}$ ).

\section{Solve The UXILIARY VARIABles By The Block RATE}

Calculate the value of the auxiliary variables use the method which is in the link without wavelength converter, solve the block rate by two methods.

\section{A. Solution Method 1}

So, first of all, we're going to use $\mathrm{C}_{\mathrm{k}}$ to figure out what the block rate is. The probability that use the determined wavelength passes smoothly between all nodes can be solved. Results are as follows.

$$
P_{\lambda}=\left(1-C_{k}\right)^{H}
$$

$C_{k}$ is the probability of k paths where the wavelength is a certain value $(\lambda)$ and between two certain nodes(i, $\mathrm{j})$ all being blocked. $\mathrm{H}+1$ represents the number of nodes that need to pass through for transmission.

So the probability that a single wavelength cannot be transmitted successfully is called the blocking rate can be solved .Results are as follows:

$$
C_{0}=1-P_{\lambda}=1-\left(1-C_{k}\right)^{H}
$$


So the probability that all wavelengths cannot be transmitted successfully is called the blocking rate can be solved .Results are as follows:

$$
C_{1}=\left(1-P_{\lambda}\right)^{w}=\left(1-\left(1-C_{k}\right)^{H}\right)^{w}
$$

$\mathrm{w}$ represents the number of wavelength categories contained in each link

\section{B. Solution Method 2}

Let's solve the block rate the other way, We break up the whole link network into countless point-to-point connections. In this way, we first need to consider: network load on certain connection ( denote $\mathrm{asL}_{\mathrm{ij}}$ ) :

$$
L_{i j}=\frac{\sum_{s, d=0} R_{S d}}{k}
$$

$\left\{R_{s d}\right\}$ is represented call arrival rate per unit time from node $\mathrm{s}$ to node $\mathrm{d}$, When $\mathrm{s}$ is equal to $\mathrm{d} R_{s d}=0$, therefore $\sum_{s, d=0} R_{s d}$ represent all links in the total arrival rate. $\mathrm{k}$ is the number of links between two nodes.

We can derive from these inferences that in the case of just using some certain wavelength, he probability of successful response to the call is $\overline{L_{\imath \jmath}}$. In turn, we can obtain that the probability that the optical path is successfully established when a signal is applied to transmit from $\mathrm{i}$ to $\mathrm{j}$ is:

$$
\mathrm{f}(\mathrm{i}, \mathrm{j})=1-\left(1-\sum_{i, j=1}^{w} \overline{L_{l \jmath}}\right)^{k}
$$

Then the light path cannot be successfully established, that also is the probability of blocking is:

$$
C_{i j}=1-f(i, j)
$$

So we can then figure out what the block rate is:

$$
C_{1}=\frac{\sum_{s, d=1} R_{S d} C_{i j}}{\sum_{s, d=0} R_{s d}}
$$

\section{Simultaneous Exploration}

Simultaneous equation:

$$
\frac{\sum_{s, d=1} R_{S d} C_{i j}}{\sum_{s, d=0} R_{s d}}=C_{1}=\left(1-P_{\lambda}\right)^{w}=\left(1-\left(1-C_{k}\right)^{H}\right)^{w}
$$

Therefore, we can calculate the expression of $C_{k}$ :

$$
C_{k}=1-\sqrt[H]{\left(1-\sqrt[w]{\frac{\sum_{s, d=1} R_{s d} C_{i j}}{\sum_{s, d=0} R_{S d}}}\right)}
$$

\section{Solve The Packet Loss Rate In LinKs Without WAVELENGTH CONVERTER}

After Before starting, we should first test the specific transmission efficiency of the link. The performance of multiple optical fibers is measured and averaged to obtain data. The packet loss rate of each wavelength was recorded as $\left\{P_{1}, P_{2}, P_{3} P_{4}, P_{5} \ldots \ldots . P_{w}\right\} \quad$. To correspond $\left\{\lambda_{1}, \lambda_{2}, \lambda_{3} \lambda_{4}, \ldots \ldots . \lambda_{w}\right\}$ these several wave length. And to make it easier to write, we're going to rearrange the probabilities of these packets in order from small to large. Get a new set of data $\left\{P_{1}, P_{2}, P_{3} P_{4}, P_{5} \ldots \ldots P_{w}\right\}$. and $\left\{\lambda_{1}, \lambda_{2}, \lambda_{3} \lambda_{4}, \ldots \ldots . \lambda_{w}\right\}$.

\section{A. Allocation Algorithm Analysis}

In a network without wavelength converters, Information is transmitted at a certain wavelength. on this occasion, It is necessary that we first define the most reasonable method of wavelength assignment. We analyze the implementation of the wavelength selection algorithm. First, the packet loss rate of this wavelength must be the lowest under the current situation. But at the same time, we are forced to use links with higher packet loss rates, as the higher ranked wavelengths may have been consumed by previous transmissions. Therefore, the main content of this algorithm is to select the wavelength with the lowest packet loss rate from the current available wavelength to complete the transmission ${ }^{[3]}$.

\section{B. The Specific Calculation}

In this algorithm, we first analyze the probability of each wavelength being enabled. The probability that all the transmission channels of other wavelengths prior to the $\lambda_{i}$ wavelength between the two nodes are occupied is:

$$
C_{i}=\left(C_{k}\right)^{i-1}\left(1-C_{k}\right)
$$

The probability that the transmission channel which precedes the other wavelength of wave length $\lambda_{i}$ between two nodes is not occupied is:

$$
\overline{C_{i}}=1-\left(C_{k}\right)^{i-1}\left(1-C_{k}\right)
$$

Therefore, we can obtain the probability that the transmission channel of all the nodes is occupied before the wavelength of $\lambda_{i}$ :

$$
C_{i}=\sum_{j=1}^{H-1}\left(\left(C_{k}\right)^{i-1}\left(1-C_{k}\right)\right)^{j}\left(1-\left(C_{k}\right)^{i-1}\left(1-C_{k}\right)\right)^{H-j}
$$

$$
\begin{gathered}
C_{i}= \\
{\left[\left(\left(C_{k}\right)^{i-1}\left(1-C_{k}\right)\right)+1-\left(C_{k}\right)^{i-1}\left(1-C_{k}\right)\right]^{H}-} \\
\left(1-\left(C_{k}\right)^{i-1}\left(1-C_{k}\right)\right)^{H}
\end{gathered}
$$

Transformation from (12) to (13) use knowledge of binomial theorem. ${ }^{[4]}$

Therefore, the packet loss rate is:

$$
D_{r}=\sum_{i=1}^{w} C_{i} * P_{i}
$$

\section{Solve The Packet Loss Rate With Wavelength CONVERTER LINK}

When we analyze the packet loss rate, we can convert the calculation of the packet loss rate in the whole transmission process into partial packet loss rate. Separate by the wavelength converter in the link, ensuring that each separate part has no 
wavelength converter in between so that each small segment can be processed into a simple part without wavelength converter. So the number of nodes that have each segment going through is $\left\{H_{1}+1, H_{2}+1, H_{3}+1, H_{4}+1 \ldots \ldots \ldots \ldots \ldots H_{m}+1\right\}($ The number of segments divided is denoted as $\mathrm{m}$ ) In this way, we can obtain the packet loss rate of the whole link. Then the probability that the wavelength occupied before $\lambda_{\mathrm{i}}$ in the j segment is:

$$
\begin{gathered}
C_{i_{j}}=\left[\left(\left(C_{k}\right)^{i-1}\left(1-C_{k}\right)\right)+1-\left(C_{k}\right)^{i-1}\left(1-C_{k}\right)\right]^{H_{j}}- \\
\left(1-\left(C_{k}\right)^{i-1}\left(1-C_{k}\right)\right)^{H_{j}}
\end{gathered}
$$

Therefore, the packet loss rate of each small segment is:

$$
D_{r_{j}}=\sum_{i=1}^{w} C_{i_{j}} * P_{i}
$$

The packet loss rate can be obtained as follows:

$$
D_{r}=\prod_{j=1}^{m} D_{r_{j}}
$$

\section{TEST The Results}

The calculated results obtained by the algorithm in this paper are compared with the actual results to solve the variance. The results are as figure I:

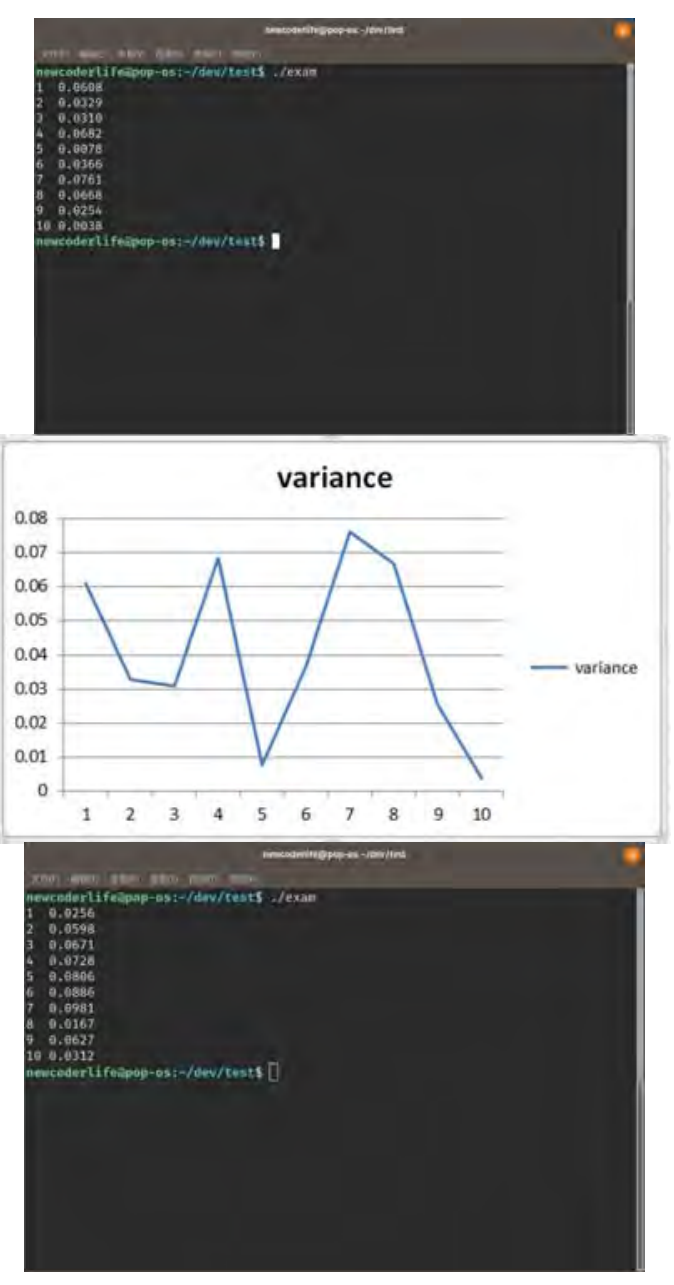

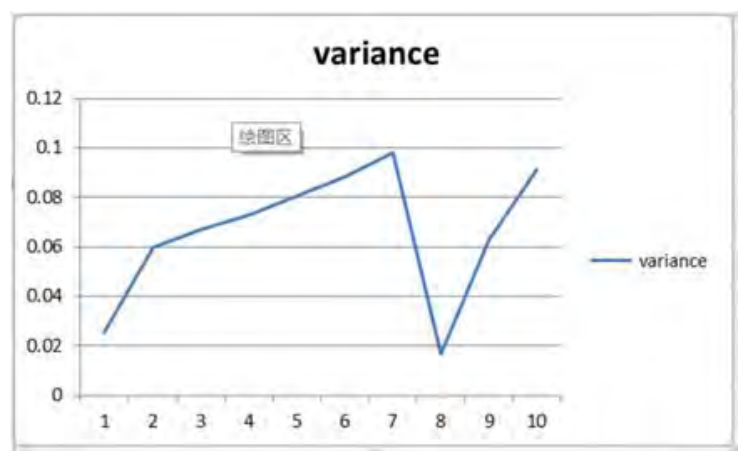

FIGURE I. RESULTS OF TWO SIMULATION EXPERIMENTS.

\section{REFERENCES}

[1] [US] James F. Kurose [US]Keith W. Ross ,Computer network (edition 5), China Machine Press

[2] Zhongyuan Jiang, Mangui Liang, analysis and optimization of transmission capacity of complex network,9787512124745.

[3] [Singapore]Tracey Ho [Aus]Desmond S. Lun ,Introduction to network coding China Machine Press

[4] Department of mathematics, tongji university, Advanced mathematics (7th edition),Higher Education Press 